\title{
The identification of quantitative trait loci that control the paternal inheritance of a mitochondrial plasmid in rapeseed (Brassica napus L.)
}

\author{
Masao Oshima ${ }^{1}$ and Hirokazu Handa ${ }^{1,2 *}$ \\ ${ }^{1}$ Graduate School of Life and Environmental Sciences, University of Tsukuba, 1-1-1, Ten-noh-dai, \\ Tsukuba 305-8572, Japan \\ ${ }^{2}$ Plant Genome Research Unit, National Institute of Agrobiological Sciences, \\ 2-1-2, Kan-non-dai, Tsukuba 305-8602, Japan
}

(Received 20 November 2011, accepted 30 January 2012)

\begin{abstract}
Some varieties of Brassica napus (rapeseed) and B. rapa contain a liner mitochondrial plasmid that is unique in that it can be inherited from the male parent through the pollen. We found that two rapeseed cultivars, Norin 16 and Westar, showed different rates of plasmid inheritance from the paternal parent $(78.8 \%$ and $27.5 \%$, respectively). To identify nuclear genes controlling the inheritance of the plasmid, we carried out quantitative trait locus (QTL) analyses using $\mathrm{F}_{2}$ populations derived from a cross between these two cultivars. The $\mathrm{F}_{1}$ plants transmitted the plasmid from the paternal plant at a frequency of approximately 60\%; the transmission rates of the $\mathrm{F}_{2}$ lines varied greatly, from 0 to $100 \%$, with an average of $68.2 \%$. A genetic map was constructed based on the segregation of 175 loci in the $102 \mathrm{~F}_{2}$ plants. A total of 22 linkage groups were obtained, all of which could be assigned to the 19 rapeseed chromosomes. The total map length was 1374.7 $\mathrm{cM}$, with an average distance of $7.9 \mathrm{cM}$ between the markers. We found that three quantitative trait loci for plasmid paternal transfer, $q P p t 1, q P p t 2$ and $q P p t 3$, located on chromosomes A5, C2 and C9, respectively, were significantly linked to the transmission frequency, whose the logarithm of odds (LOD) score were 4.97, 3.49 and 3.57, respectively. Their explained phenotypic variances were $25.0 \%$, $22.2 \%$ and $37.1 \%$, respectively. These results suggest that the paternal inheritance of the mitochondrial plasmid is controlled by a relatively small number of nuclear genes.
\end{abstract}

Key words: mitochondria, paternal inheritance, plasmid, QTLs, rapeseed

\section{INTRODUCTION}

Several higher plants have a variety of smaller mitochondrial DNA molecules, so called plasmid or plasmidlike DNA in addition to the main mitochondrial genome (reviewed in Handa, 2008). The mitochondrial plasmid found in rapeseed (Brassica napus L.) is a linear molecule of $11,640 \mathrm{bp}$ that encodes six open reading frames (ORFs) (Handa et al., 2002). All of these ORFs are transcribed, and at least three of the six ORFs are translated. Although the functions of these ORFs are unclear, based from the sequence similarity, orf5 and orf6 are predicted to encode a phage-type DNA polymerase and an RNA polymerase, respectively. These results suggest that the

Edited by Toru Terachi

* Corresponding author. E-mail: hirokazu@affrc.go.jp

Note: Supplementary materials in this article are at http:// www.jstage.jst.go.jp/browse/ggs rapeseed plasmid is able to replicate itself and to transcribe the encoded genetic information in the mitochondria. Extensive phylogenetic studies have shown that the presence of the plasmid is limited to B. rapa landraces and rapeseed varieties derived from interspecific crosses between $B$. napus and $B$. rapa, indicating that this plasmid originated from $B$. rapa and was introduced into the rapeseed mitochondria through interspecific crosses (Handa, 2007).

In higher plant species, organellar genomes (i.e., mitochondrial and chloroplast genomes) usually exhibit uniparental transmission from the maternal parent to the progeny. However, the rapeseed mitochondrial plasmid is unique in that it can be inherited through pollen (Erickson et al., 1989). This unique inheritance pattern has also observed for sugar beet mitochondrial plasmid (SaumitouLaprade et al., 1989). Although the paternal or biparental inheritance of mitochondrial genomes has been 
reported in species such as bananas, cucumber and Wisteria (Faure et al., 1994; Havey, 1997; Trusty et al., 2007), the paternal inheritance of the rapeseed mitochondrial plasmid is completely independent from the inheritance of the main mitochondrial genome, i.e., the plasmid may be inherited paternally even if the mitochondrial genome shows a strict maternal inheritance pattern. Fluorescence microscopy observation has shown that mitochondrial DNA molecules disappear during pollen development in rapeseed (Sato et al., 2004). In Arabidopsis, the active degradation of paternal mitochondria in the zygote within five hours after fertilization was observed in transgenic plants with mitochondrion-targeted fluorescent proteins (Matsushima et al., 2008). These results suggest that the plasmid has a mechanism that protects it from the usual elimination of mitochondria and/or mitochondrial DNA inherited from pollen.

The nuclear genetic background affects the rate of paternal inheritance of this plasmid (Erickson et al., 1989; Handa, 2007), indicating that the paternal inheritance of the plasmid is controlled by a nuclear gene(s) involved in the transfer and maintenance of plasmid in the progeny. Although the identification of these genes is necessary to understand the mechanism of the paternal inheritance of the plasmid, to date, there is little information about these genes. Thus, to identify the genes that regulate the paternal inheritance of this mitochondrial plasmid, we carried out a quantitative trait loci (QTL) analysis in the $F_{2}$ populations derived from a cross between two rapeseed cultivars with different plasmid transfer rates. We also examined the relationship between QTLs and several nuclear genes that are known to affect the structure of the mitochondrial genome in higher plants.

\section{MATERIALS AND METHODS}

Plant materials Three cultivars of rapeseed (Brassica napus L.), cv. Norin 16, cv. Westar and cv. Isuzu-natane, were used in this study. Norin 16 and Westar do not carry the plasmid in their mitochondria, whereas Isuzunatane does carry the plasmid (Table 1). A total of 102 $\mathrm{F}_{2}$ plants derived from the cross between Norin 16 and Westar were used for the genetic map construction and QTL analysis.

Isolation of total DNA Total DNA was isolated from $100 \mathrm{mg}$ of mature leaf tissue using the CTAB extraction method (Murray and Thompson, 1980) and was used to detect polymorphisms between the parent cultivars and to construct the genetic map.

A simpler DNA extraction method was used for the PCR-based examination of the plasmid inheritance and mitochondrial genome type; approximately $0.5 \mathrm{mg}$ of leaf tissue was homogenized in $100 \mu \mathrm{l}$ of SNET buffer $(20 \mathrm{mM}$
Table 1. Plant materials used in this study

\begin{tabular}{|c|c|c|c|}
\hline Plant materials & $\begin{array}{l}\text { Mt genome } \\
\text { type* }\end{array}$ & Plasmid ${ }^{* *}$ & $\begin{array}{l}\text { Transfer rate of } \\
\text { the paternal } \\
\text { inheritance of } \\
\text { plasmid**** }\end{array}$ \\
\hline Norin 16 & I & - & $78.8 \%(26 / 33)$ \\
\hline Westar & I & - & $27.5 \%(11 / 40)$ \\
\hline Isuzu-natane & II & + & N.D. \\
\hline $\mathrm{F}_{1}($ Norin $16 \times$ Westar $)$ & I & \pm & $65.0 \%(13 / 20)$ \\
\hline $\mathrm{F}_{1}($ Westar $\times$ Norin 16$)$ & I & \pm & $60.0 \%(12 / 20)$ \\
\hline $\mathrm{F}_{2}($ Westar $\times$ Norin 16$)$ & I & \pm & $68.2 \% * * * *$ \\
\hline \multicolumn{4}{|c|}{$\begin{array}{l}* \text { based on the classification by Handa et al. (1990). } \\
\text { ** +, with plasmid; -, without plasmid; } \pm \text {, with or without } \\
\text { plasmid. } \\
\text { *** } \\
\text { when they were crossed with Isuzu-natane. Real } \\
\text { numbers of plasmid-bearing plants/examined plants } \\
\text { are shown in parentheses. }\end{array}$} \\
\hline
\end{tabular}

Tris-HCl [pH 8.0], $5 \mathrm{mM}$ EDTA, $400 \mathrm{mM} \mathrm{NaCl}$ and $0.3 \%$ SDS) and $2 \mu \mathrm{l}$ of proteinase $\mathrm{K}(10 \mathrm{mg} / \mathrm{ml})$; the samples were incubated at $55^{\circ} \mathrm{C}$ for two hours, and $200 \mu \mathrm{l}$ of ethanol was then added to precipitate the total DNA.

Examination of the paternal inheritance of the plasmid and the mitochondrial genome type $\mathrm{F}_{2}$ plants derived from the cross between Norin 16 and Westar were pollinated with Isuzu-natane-derived pollen, which carries the mitochondrial plasmid. Eighteen progeny from the cross of each $\mathrm{F}_{2}$ plant and Isuzu-natane were examined for the presence of the plasmid and the mitochondrial genome type by the PCR analysis. The sequences of the primer pairs used to determine the presence of the plasmid and to conduct the mitochondrial genome typing are shown in Table 2. Total DNA isolated by the simplified extraction method was used as a template. The PCR was performed with GoTaq DNA polymerase (Promega, CA, USA) using a MyCycler thermal cycler (Bio-Rad, CA, USA) or a GeneAmp PCR System 9700 (Applied Biosystems, CA, USA). The amplification conditions were $5 \mathrm{~min}$ at $94^{\circ} \mathrm{C}$, followed by 30 cycles of $30 \mathrm{sec}$ at $94^{\circ} \mathrm{C}, 30 \mathrm{sec}$ at $55^{\circ} \mathrm{C}$ and $1 \mathrm{~min}$ at $72^{\circ} \mathrm{C}$ and a final extension of $5 \mathrm{~min}$ at $72^{\circ} \mathrm{C}$.

SSR amplification and detection of polymorphisms The following previously published simple sequence repeat (SSR) markers were used in this study: B.n. (Kresovich et al., 1995; Szewc-McFadden et al., 1996); BnEMS/BnGMS/BoGMS/BrGMS (Cheng et al., 2009; Fan et al., 2010; Li et al., 2010; Xu et al., 2010); BRAS (Piquemal et al., 2005); BRMS (Suwabe et al., 2006); CB (Piquemal et al., 2005; Radoev et al., 2008); FITO (Iniguez-Luy et al., 2008); KBr (Hatakeyama et al., 2010; Kakizaki et al., 2011); MD/MR (Radoev et al., 2008) and $\mathrm{Na} / \mathrm{Ni} / \mathrm{Ol}$ (Piquemal et al., 2005; Radoev et al., 2008).

Furthermore, novel SSR markers were designed based 
Table 2. Primer sequences that were used in this study to determine the presence of the plasmid and the mitochondrial genome type or to amplify the rapeseed homologs of Arabidopsis Msh1, RecA3 and OSB1

\begin{tabular}{|c|c|c|}
\hline Name & Sequence (5' - 3') & References \\
\hline \multicolumn{3}{|c|}{ For the plasmid presence } \\
\hline P2-F & 5'CCATGGTCCGTTCTTATTGGAAAGATCAC-3' & AB073400, Handa (2007) \\
\hline $\mathrm{P} 2-\mathrm{R}$ & 5'GTTCTTCCATGGAATATGGTAATATTTGG-3' & AB073400, Handa (2007) \\
\hline P3-F & 5'CCATGGATGATTCGACCCTCTTTCATAAG-3' & AB073400, Handa (2007) \\
\hline P3-R & 5'-CCCATGGAATAGAATGCCTTCTCCAATTC-3' & AB073400, Handa (2007) \\
\hline \multicolumn{3}{|c|}{ For the mitochondrial genome typing } \\
\hline GC-F & 5'GTGGGGCTGCAGGTACTATGGATC-3' & AP006444, Handa (2007) \\
\hline GC-R & 5'-GATTCGAAGAGAGCCAAGGTGAAC-3' & AP006444, Handa (2007) \\
\hline GD-F & 5'TTGCAGCTACGTTCCCTCCCTTTG-3' & AP006444, Handa (2007) \\
\hline GD-R & 5'- ATGCTTCGACGATTTGACTCGTGC-3' & AP006444, Handa (2007) \\
\hline \multicolumn{3}{|c|}{ For the amplification of Msh1, RecA3 and OSB1 } \\
\hline BnMSH1F & 5’-CCTCCTGCTTATGATATTGCTCTG-3’ & \multirow{2}{*}{ ES941750, EV099786, EX136750, GR442237 } \\
\hline BnMSH1R & 5'-CTATATGGATTCCCTTAACGCGAC-3' & \\
\hline BnRecA3F & 5'TCACTCAACCTTGATCTAGCTCTAG-3' & \multirow{2}{*}{$\begin{array}{l}\text { CD836046, CN828881, CN828882, EE442513, } \\
\text { EE526368, ES918040, ES934272, EV020087, EX039353 }\end{array}$} \\
\hline BnRecA3R & 5'-CGACCAAAGTGAATCCCCAATTCAG-3' & \\
\hline BnOSB1F & 5'-GCTTCTTCTGCTATTCCCAAACCTC-3' & \multirow{2}{*}{ EX126020 } \\
\hline BnOSB1R & 5'-GGATCCTTTTAATCAATGTGGATTC-3' & \\
\hline
\end{tabular}

on B. rapa BAC sequences (B. rapa Genome Sequencing Project, http://www.brassica-rapa.org/BRGP/index.jsp), Arabidopsis BAC sequences (The Arabidopsis Information Resource, http://www.arabidopsis.org/), B. oleracea BAC sequences (Gao et al., 2004, 2005, 2006), using Imperfect SSR Finder Version 1.0 (Stieneke and Eujayl, 2007; http://ssr.nwisrl.ars.usda.gov/) and Primer3 (Rozen and Skaletsky, 2000; http://frodo.wi.mit.edu/primer3/).

Total DNA extracted using the CTAB method from the parental and $\mathrm{F}_{2}$ plants was used as the templates in the PCR amplification of fragments containing each SSR marker. The PCR was performed using the following protocol: $5 \mathrm{~min}$ at $94^{\circ} \mathrm{C}$, followed by 38 cycles of $30 \mathrm{sec}$ at $94^{\circ} \mathrm{C}, 30 \mathrm{sec}$ at $55^{\circ} \mathrm{C}$ and $30 \mathrm{sec}$ at $72^{\circ} \mathrm{C}$. Polymorphisms within the PCR products were analyzed with a highefficiency genome scanning (HEGS) system (Nihon Eido, Japan) as described by Hori et al. (2003).

To map the rapeseed homologs of three Arabidopsis genes, Msh1 (Abdelnoor et al., 2003), RecA3 (Shedge et al., 2007) and OSB1 (Zaegel et al., 2006), Brassica cDNA sequences registered in the DDBJ/EMBL/GenBank databases were screened using a BLAST search with the cDNA sequences of these genes as the queries. Primers were then designed based on the sequence information for the putative rapeseed homologs. The sequences of the primer pairs for the rapeseed homologs of $M s h 1, R e c A 3$ and $O S B 1$ are provided in Table 2. A fragment of each of these rapeseed homologs was amplified by PCR, and the amplified products were sequenced. The PCR amplification was performed under the following conditions: $5 \mathrm{~min}$ at $94^{\circ} \mathrm{C}$, followed by 30 cycles of $30 \mathrm{sec}$ at $94^{\circ} \mathrm{C}$,
$30 \mathrm{sec}$ at $55^{\circ} \mathrm{C}$ and $1 \mathrm{~min}$ at $72^{\circ} \mathrm{C}$. The sequences of the PCR fragments from Norin 16 and Westar were compared to detect any polymorphisms, and a cleaved amplified polymorphic sequence (CAPS) assay was designed based on this comparison.

Construction of the map and QTL analysis A total of $102 \mathrm{~F}_{2}$ plants were genotyped for each of the polymorphic SSRs identified in the parents, and this information was used for the construction of the genetic map. Linkage analysis was performed using the Antmap program ver. 1.2 (Iwata and Ninomiya, 2006), and a graphical presentation of the linkage map was drawn in MapChart 2.1 (Voorrips, 2002). The QTL analysis was performed by composite interval mapping using Windows QTL Cartographer version 2.5 (Wang et al., 2007; http://statgen. ncsu.edu/qtlcart/WQTLCart.htm) with the forward and backward regression. A logarithm of odds (LOD) score threshold of 2.5 was determined by computing 1,000 permutations.

\section{RESULTS}

Rate of plasmid transmission through pollen The transmission rate of the plasmid through pollen was evaluated for the two parental cultivars, Norin 16 and Westar, neither of which normally carry plasmids in their mitochondria. We crossed these two cultivars with the plasmid-bearing cv. Isuzu-natane as the paternal parent, and we then analyzed for the presence of the plasmid and the mitochondrial genome type in the resulting progeny. 
The transfer rates of the plasmid to the progeny for Norin 16 and Westar were $78.8 \%$ and $27.5 \%$, respectively (Table 1). This difference in rates between the two cultivars might reflect the differences in their nuclear genomes.

To investigate the paternal inheritance of the plasmid further, we examined the plasmid transfer rates for the $F_{1}$ and $F_{2}$ plants from the cross between Norin 16 and Westar using test crosses with Isuzu-natane. In addition, we analyzed the mitochondrial genome types of progeny from the $F_{2}$ plant $x$ Isuzu-natane and the $F_{1}$ plant $x$ Isuzu-natane to confirm the maternal inheritance pattern of the mitochondrial genome. The mitochondrial genomes of rapeseed have been classified into 2 major types, type I and type II (Handa et al., 1990). Norin 16 and Westar have type I mitochondria, whereas Isuzunatane has type II. If paternal inheritance of the main mitochondrial genome occurs, then the progeny from the crosses with Isuzu-natane pollen should have type II mitochondria derived from Isuzu-natane.

Twenty plants from the crosses between the $\mathrm{F}_{1}$ plants and Isuzu-natane were chosen randomly for PCR analyses. The PCR was carried out using the P2, P3, GC and GD primer pairs to amplify the coding regions of orf 2 and $\operatorname{orf} 3$ in the plasmid, the region around exon $\mathrm{c}$ of nad5 and the region downstream of $\operatorname{cox} 1$ in the mitochondrial genome, respectively, to evaluate the plasmid inheritance and mitochondrial genome type. All of the progeny from the crosses between the reciprocal $\mathrm{F}_{1}$ plants (Norin $16 \times$ Westar and Westar $\times$ Norin 16) and Isuzu-natane had type I mitochondria (Table 1), and the plasmid transfer rates of the reciprocal $\mathrm{F}_{1}$ plants (Norin $16 \times$ Westar and Westar $\times$ Norin 16 ) were $65.0 \%$ and $60.0 \%$, respectively (Table 1).

Progeny of $87 \mathrm{~F}_{2}$ plant crossed with Isuzu-natane were examined for the presence of the plasmid and mitochondrial genome type by PCR analysis. All of the progeny had type I mitochondria (Table 1). The plasmid transfer rates for the $\mathrm{F}_{2}$ individuals, deduced from the test cross with Isuzu-natane, varied greatly, ranging from $0 \%$ to $100 \%$; however, individuals with higher rates (equivalent to Norin 16) were more frequent than the individuals with lower rates (similar to Westar) (Fig. 1). The average transfer rate was $68.2 \%$, a value that was slightly higher than that of the reciprocal $\mathrm{F}_{1}$ plants (Table 1 and Fig. 1).

Polymorphism survey and construction linkage map To develop novel markers, SSRs were screened based on sequence information from $B$. rapa BAC sequences, Arabidopsis BAC sequences and B. oleracea BAC sequences. As a result, 232 primer sets for the amplification of new SSRs were designed (Table 3 and Supplementary Table S1). In addition to the newly developed primer sets, we also used 1915 published primer sets; $456 \mathrm{KBr}, 326$ BRMS, $169 \mathrm{CB}, 159$ BoGMS,

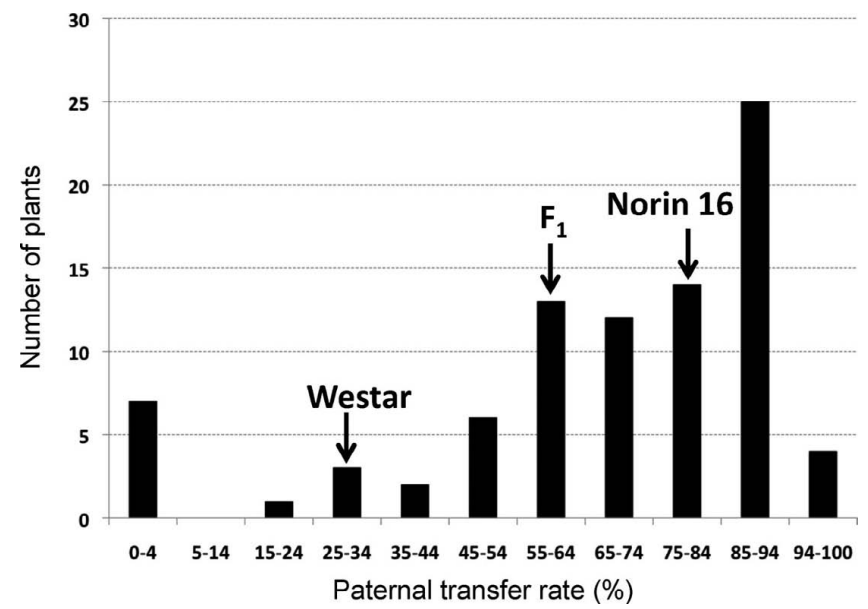

Fig. 1. Frequency distribution of the paternal plasmid transfer rates in the $\mathrm{F}_{2}$ plants derived from the cross between Westar and Norin 16. The transfer rates were calculated using eighteen progeny from each cross of the $\mathrm{F}_{2}$ plant $\mathrm{x}$ Isuzu-natane (see details in MATERIALS AND METHODS). The rates of the parent cultivars and their $\mathrm{F}_{1}$ progeny are indicated with arrows.

Table 3. Novel SSR and CAPS markers introduced in this study*

\begin{tabular}{cccc}
\hline \hline Maker name & No. of markers & Type of markers & Origin \\
\hline BrMS_UT & 126 & SSR & B. rapa \\
BoMS_UT & 31 & SSR & B. oleracea \\
AtMS_UT & 75 & SSR & A. thaliana \\
BnMSH1 & 1 & CAPS & B. napus \\
BnOSB1 & 1 & CAPS & B. napus \\
\hline
\end{tabular}

* Precise information for each marker is shown in Supplementary Table S1.

132 BnGMS, $100 \mathrm{Na}, 100$ Ni, 95 Ol, 89 Ra, 77 BrGMS, 75 BRAS, 50 FITO, 30 BnEMS, $21 \mathrm{MR}, 19 \mathrm{MD}$ and 17 B.n. In total, 2147 SSR primer sets were tested for polymorphism between Norin 16 and Westar, 209 of which $(9.7 \%)$ showed polymorphisms. The genetic map was constructed based on the segregation of 184 loci using the $102 \mathrm{~F}_{2}$ progeny from the cross between Westar and Norin 16. A total of 173 markers were placed in 22 linkage groups, with a total distance of $1374.7 \mathrm{cM}$ (Supplementary Fig. S1). Although three chromosomes, C3, C5 and C8, contain gaps, each linkage group could be assigned to a specific chromosome (A1-C9) using the chromosomespecific SSR markers. The largest linkage group maps to chromosome A5, consisting of 24 loci with a length of $157.2 \mathrm{cM}$, whereas the smallest group maps to chromosome $\mathrm{C} 7$, and contains four loci with a length of $5.0 \mathrm{cM}$ (Supplementary Fig. S1). The distances between the markers varied from 0 to $43.3 \mathrm{cM}$, with an average distance of $7.9 \mathrm{cM}$.

Several nuclear genes have been reported to affect the structure of the mitochondrial genome in Arabidopsis. Lesions in these genes, especially Msh1, RecA3 and $O S B 1$, result in increasingly irregular recombination 


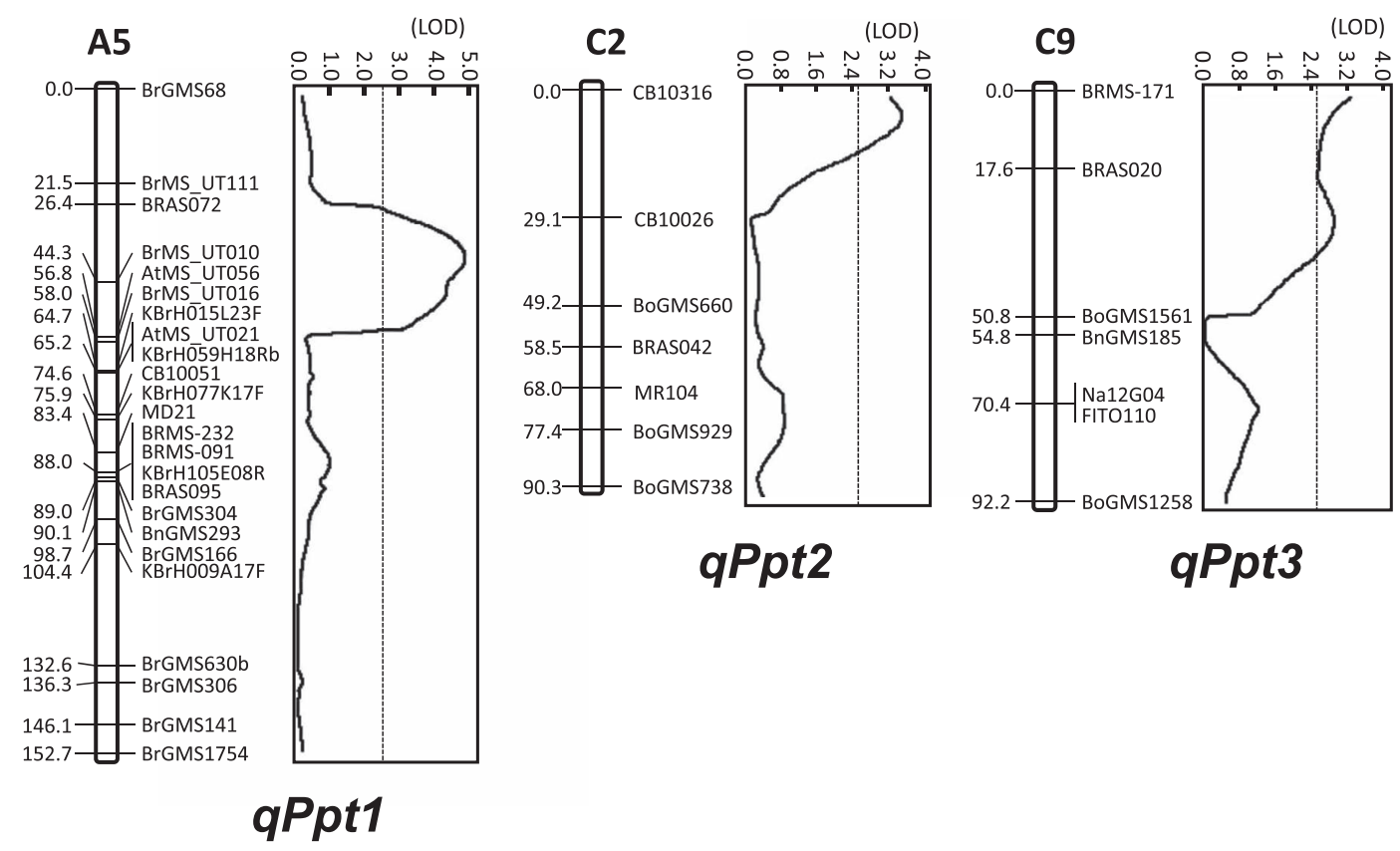

Fig. 2. Linkage maps and QTL-likelihood curves of LOD scores showing the locations of the QTLs for the paternal inheritance of the plasmid, $q P p t 1, q P p t 2$ and $q P p t 3$, on chromosomes A5, C2 and C9, respectively. Genetic distances are shown in cM on the left of the chromosome. The 2.5 LOD score threshold is indicated with a dashed line.

events (Abdelnoor et al., 2003; Shedge et al., 2007; Zaegel et al., 2006). To clarify the relationship between these genes and the inheritance of the mitochondrial plasmid through pollen, we attempted to identify these genes on our genetic map (Supplementary Fig. S1). We amplified portions of the rapeseed homologs of Msh1, RecA3 and OSB1 from Westar and Norin 16 and then compared their sequences. We found polymorphisms in $M s h 1$ and $O S B 1$, allowing us to map these two genes to chromosomes A7 and C3, respectively, as CAPS markers (Supplementary Fig. S1).

QTL analysis for the inheritance of the mitochondrial plasmid Each QTL for plasmid inheritance was detected based on the plasmid transfer rate data (Fig. 1) from $\mathrm{F}_{2}$ individuals and the genetic map constructed using the $\mathrm{F}_{2}$ population (Supplementary Fig. S1). Three QTLs showing significant LOD scores $(>2.5, \mathrm{P}<0.05)$ were detected on chromosomes A5, C2 and C9. The QTL located on chromosome A5 had the highest LOD score (4.97), and contributed $25.0 \%$ of the variance in the trait (Fig. 2, Table 4). This QTL was detected within the $31.6 \mathrm{cM}$ interval between the BRAS072 and AtMS_UT056 loci, and it was most closely linked to BrMS_UT010 (Fig. 2). This QTL was named quantitative trait locus 1 for plasmid paternal transfer 1 ( $q$ Ppt1).

The two other QTLs identified had LOD scores of 3.49 on chromosome C2 and 3.57 on chromosome C9 (Fig. 2, Table 4). The C2 QTL was located distally to CB10026 and was most closely linked to CB10316 (Fig. 2), and the C9 QTL was distal to BoGMS1561 and most closely linked
Table 4. Summary of the QTLs for the paternal inheritance of the mitochondrial plasmid

\begin{tabular}{|c|c|c|c|c|c|c|}
\hline Name & Chromosome & $\begin{array}{l}\text { Closest } \\
\text { marker }\end{array}$ & LOD & $\mathrm{R}^{2}(\%)^{*}$ & $\begin{array}{l}\text { Additive } \\
\text { effect** }\end{array}$ & $\begin{array}{c}\text { Dominance } \\
\text { effect }\end{array}$ \\
\hline$q P p t 1$ & A5 & BrMS_UT101 & 4.97 & 25.01 & 18.35 & 15.72 \\
\hline$q P p t 2$ & $\mathrm{C} 2$ & CB10316 & 3.49 & 22.19 & 17.10 & 13.60 \\
\hline$q P p t 3$ & C9 & BRAS020 & 3.57 & 37.05 & -19.90 & 22.26 \\
\hline
\end{tabular}

* proportion of the phenotypic variance explained by each QTL. ** additive effect of the Norin 16 allele.

to BRAS020 (Fig. 2). These two loci accounted for $22.2 \%$ and $37.1 \%$ of phenotypic variance (Table 4 ) and were designated $q P p t 2$ and $q P p t 3$, respectively. Norin 16 alleles of $q P p t 1$ and $q P p t 2$ and the Westar allele of $q P p t 3$ contributed to higher plasmid transfer through the pollen (Table 4).

The effects of allelic substitutions at one QTL differed depending on other QTL genotypes, although most of them were not statistically significant due to the small sample size probably (Fig. 3). The plants homozygous for the favorable alleles of both $q P p t 1$ and $q P p t 2$ loci, Norin 16 alleles for $q P p t 1$ and $q P p t 2$, showed a higher transmission rate than the plants homozygotes for the unfavorable (Westar) allele of $q P p t 1$ and favorable (Norin 16) allele of $q P p t 2$ (Fig. 3). Similar trends were observed in almost all genotype combinations of two loci with the higher frequency of plasmid transfer. On the other hand, the plants homozygous for the unfavorable alleles of both $q P p t 1$ and $q P p t 2$ loci, Westar alleles for both loci, showed a complete suppression on the paternal plasmid inheritance (Fig. 3). Other two combinations with the 

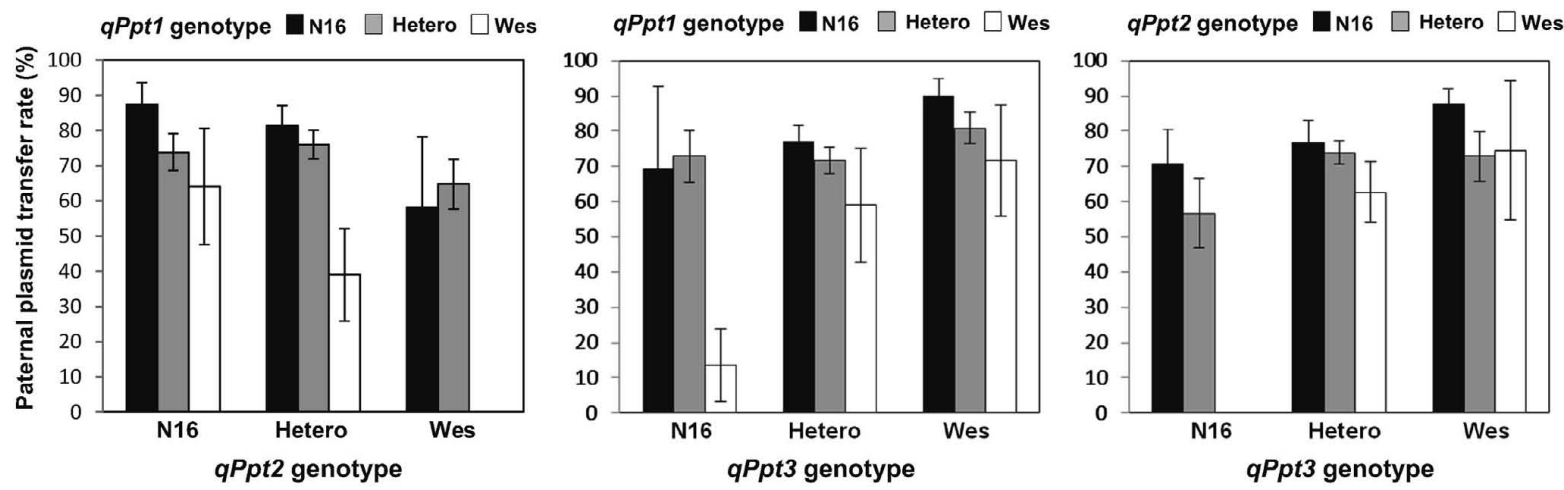

Fig. 3. Differences in the paternal plasmid transfer rates for the combinations of three QTLs. The genotypes were determined using the closely linked markers BrMS_UT101 (qPpt1), CB10316 (qPpt2) and BRAS020 (qPpt3). N16, Hetero and Wes indicate homozygous for the Norin 16 allele, heterozygous and homozygous for the Westar allele, respectively. Bars represent mean values \pm standard errors.

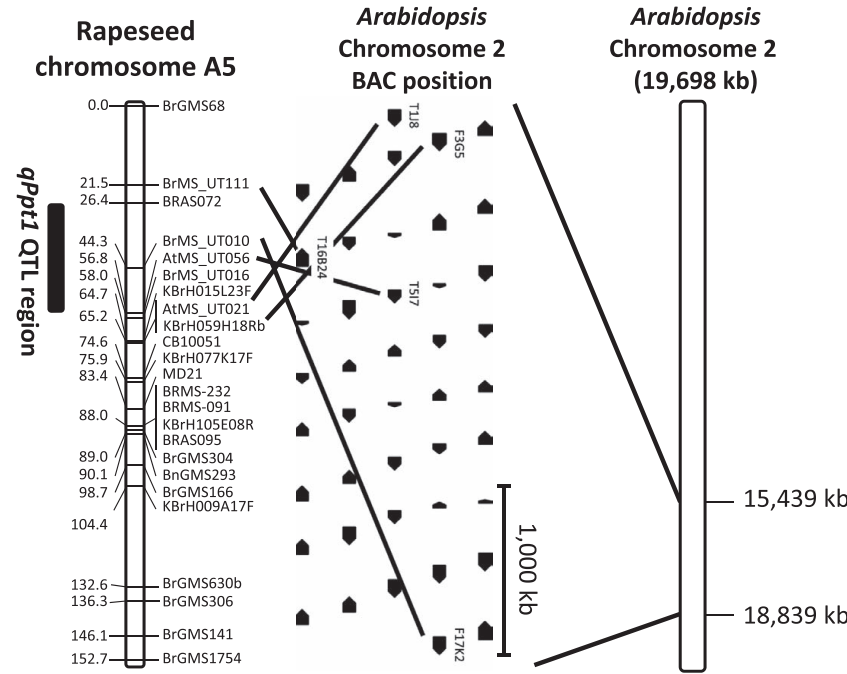

Fig. 4. Comparative analysis of the $q P p t 1$ QTL region (solid bar) on rapeseed chromosome A5 with the physical map of Arabidopsis chromosome 2. Genetic distances are shown in $\mathrm{cM}$ on the left of chromosome A5.

lower frequency of plasmid transfer also showed a complete suppression or very low rate of the plasmid transfer from the paternal plants.

Because there is well-documented synteny between Brassica species, including rapeseed and Arabidopsis, we compared our three QTL regions ( $q P p t 1, q P p t 2$ and $q P p t 3$ ) with the Arabidopsis genome sequence to obtain more precise information for the fine mapping of the QTLs. Our comparative analysis revealed that the $q P p t 1$ region aligned with the genomic region from $15,439 \mathrm{~kb}$ to $18,839 \mathrm{~kb}$ of Arabidopsis chromosome 2 (Acc. No. CP002685) (Fig. 4). Although the $q P p t 1$ region is still too large to mine the causal gene for this locus using the information of syntenic region in Arabidopsis, these are very useful for further mapping studies and were partially used for the design of the novel markers in this study.

\section{DISCUSSION}

Our phenotypic evaluation of paternal inheritance showed that the $\mathrm{F}_{1}$ plants derived from a cross between Norin 16, the cultivar with the higher transfer rate, and Westar, the cultivar with the lower rate, showed an intermediate rate of paternal inheritance. The $F_{2}$ progeny revealed a continuous distribution for the paternal inheritance of the plasmid, and transgressive segregation was observed compared with the phenotypes observed in the parental cultivars (Fig. 1). This type of continuous trait is often controlled by multiple genes or by a few individual genes. Therefore, a QTL analysis of the paternal inheritance of the plasmid was performed to determine how many genes are involved in the paternal inheritance of the plasmid in rapeseed and their chromosomal locations.

Composite interval mapping identified three QTLs, $q P p t 1, q P p t 2$ and $q P p t 3$, with significant effects on plasmid inheritance, located on chromosomes A5, C2 and C9, respectively (Fig. 2, Table 4). The LOD scores and the phenotypic variances explained by each QTL were relatively similar; the LOD scores ranged from 4.97 for $q P p t 1$ to 3.49 for $q P p t 2$, and the phenotypic variance ranged from $22.2 \%$ for $q P p t 2$ to $37.1 \%$ for $q P p t 3$ (Table 4). In all of the detected QTLs, the additive effects were nearly identical to their dominance effects, indicating that these QTLs are nearly completely dominant. We detected no clear epistasis among these QTLs (Fig. 3). These results indicate that these QTLs likely represent genes with major functions that are independent of each other. However, we observed extremely low transfer rates in plants homozygous for the unfavorable alleles of any combinations of two QTLs (Fig. 3). There is a possibility that a genetic interaction(s) occurs in the combination of specific alleles for these QTLs.

Previously, we suggested that the nuclear genome rather than the mitochondrial genome was responsible for 
the paternal transmission of the plasmid to progeny (Handa, 2007). In this study, we detected three nuclear QTLs that control plasmid transmission, yet their functions remain unclear. Although our present knowledge about the mechanism of plasmid transfer through pollen is very limited, at least three steps are postulated for plasmid transfer from the paternal mitochondria to the mitochondria of progeny: 1) escape from degradation, 2) integration into the progeny mitochondria and 3) stable maintenance in progeny plants.

Cytological observations of mature pollen grains by DAPI fluorescence microscopy has revealed a close correlation between the presence or absence of mitochondrial DNA in generative and sperm cells and the biparental or maternal inheritance of mitochondria determined in previous genetic analyses (Kuroiwa et al., 1993; Nagata et al., 1999; Nagata, 2010). Thus, DNA degradation during pollen development is a key factor for the maternal inheritance of the mitochondrial genome in higher plants. Actually, the gene for the $\mathrm{Mg}^{2+}$-dependent exonuclease which is highly active in developing pollen was recently found in Arabidopsis, although its biological function and relationship to the maternal inheritance are currently unclear (Matsushima et al., 2011). Previously, we observed mitochondrial DNA degradation during pollen development, consistent with genetic studies showing that the mitochondrial genome of rapeseed is inherited maternally (Sato et al., 2004). For the mitochondrial plasmid to be transmitted through the pollen, the plasmid DNA must contain some mechanism allowing it to escape from degradation during pollen development. However, the three QTLs detected in this study cannot be involved in this step: we used only one cultivar, Isuzu-natane, as the paternal plant, so there can be no differences in pollen development.

Transmission of sperm mitochondria from pollen into the egg and central cells has been reported in tobacco ( $\mathrm{Yu}$ et al., 1994) and Arabidopsis (Matsushima et al., 2008). If the plasmid could escape degradation, the paternal mitochondria that reach the egg cells would contain the plasmid DNA molecules. After that, the plasmid must be integrated into the maternal mitochondria. There are two hypotheses for how this step might occur: 1) the paternal and maternal mitochondria could fuse, or 2) the plasmid DNA could escape from the paternal mitochondria and then integrate into the maternal mitochondria. In plant cells, mitochondria are very dynamic organelles that undergo frequent fusion and fission events, enabling intermitochondrial exchange and recombination of mtDNA (Logan, 2006, 2010). Although the mitochondrial fission apparatus is generally conserved in a wide range of organisms, including higher plants, essentially nothing is known about the mechanism controlling mitochondrial fusion in plants. However, it has been reported that the linear mitochondrial plasmid, $\mathrm{mF}$, promotes mitochondrial fusion during the early stages of plasmodium formation in the slime mold, Physarum polycephalum (Sakurai et al., 2004). It will be very interesting to investigate the possible relationship between the mitochondrial fusion and the paternal plasmid inheritance, and its relationship with the QTLs detected in this study.

Isolated plant mitochondria are competent for DNA uptake in vitro (Koulintchenko et al., 2003, 2006). In fact, we observed the efficient uptake of the plasmid into isolated turnip mitochondria and found that terminal inverted repeats are necessary for the uptake of the plasmid (Ibrahim et al., 2011). If the three QTLs detected in this study are involved in this step, they may function as factors regulating mitochondrial fusion at fertilization or as factors promoting DNA uptake and/or designating the DNA molecule for mitochondrial uptake. Presently, we do not understand the principles of these two events, mitochondrial fusion and mitochondrial DNA uptake, fully enough to make a definitive conclusion.

The third possibility is that the QTLs encode factors that are not directly involved in the transfer of the plasmid to the mitochondria but rather in the stability of the plasmid molecules in the mitochondria of the progeny. Factors that are known to be required for the stable maintenance of the mitochondrial genome structure include Msh1, RecA3 and OSB1 in Arabidopsis (Abdelnoor et al., 2003; Shedge et al., 2007; Zaegel et al., 2006); therefore, it is possible that one or more of the three QTLs identified in this study will relate to these genes. We successfully mapped the rapeseed homologs of Arabidopsis Msh1 and OSB1 (Supplementary Fig. S1); rapeseed $M s h 1$ and $O S B 1$ were located on chromosomes A7 and C3, respectively, although they were not associated with any of the QTLs in this study. This result indicates that our QTLs are different from Msh1 and OSB1. However, this result does not exclude the possibility that the QTLs regulate the stability of the plasmid molecules in the progeny mitochondria. On the other hand, quantitative control is also important for the stability of the plasmid molecules in the mitochondria. Although we do not have so much information for this subject in plants, it is recently reported that the copy numbers of individual genes are controlled at the level of sub-genomic molecules (Preuten et al., 2010). Because the plasmid and main genome are quite different in terms of their molecular nature and behavior in vivo, it would not be surprising if its maintenance in mitochondria is under the control of different genes.

Our QTL analysis revealed that the paternal inheritance of the plasmid is not controlled by many genes with small effects but rather by a few genes with major functions. This result is very important for future work in elucidating the overall mechanism of the paternal inheritance of mitochondrial plasmids. Further experi- 
ments for the fine mapping of the QTLs and exploring candidate genes by comparing synteny with the Arabidopsis genome will clarify the functional roles of the QTLs detected in this study.

We are grateful to Dr. S. Matsumoto for his kind help with genetic mapping and providing the SSR marker information for the Brassica species. We thank Drs. H. Kawahigashi and F. Kobayashi for their valuable suggestions. This work was supported in part by a Grant-in-Aid for challenging Exploratory Research from the Japan Society for the Promotion of Science (No. 22658002).

\section{REFERENCES}

Abdelnoor, R. V., Yule, R., Elo, A., Christensen, A. C., MeyerGauen, G., and Mackenzie, S. A. (2003) Substoichiometric shifting in the plant mitochondrial genome is influenced by a gene homologous to MutS. Proc. Natl. Acad. Sci. USA 100, 5968-5973.

Cheng, X., Xu, J., Xia, S., Gu, J., Yang, Y., Fu, J., Qian, X., Zhang, S., Wu, J., and Liu, K. (2009) Development and genetic mapping of microsatellite markers from genome survey sequence in Brassica napus. Theor. Appl. Genet. 118, 1121-1131.

Erickson, L., Kemble, R., and Swanson, E. (1989) The Brassica mitochondrial plasmid can be sexually transmitted. Pollen transfer of a cytoplasmic genetic element. Mol. Gen. Genet. 218, 419-422.

Fan, C., Cai, G., Qin, J., Li, Q., Yang, M., Wu, J., Fu, T., Liu, K., and Zhou, Y. (2010) Mapping of quantitative trait loci and development of allele-specific markers for seed weight in Brassica napus. Theor. Appl. Genet. 121, 1289-1301.

Faure, S., Noyer, J. L., Carreel, F., Horry, J. P., Bakry, F., and Lanaud, C. (1994) Maternal inheritance of chloroplast genome and paternal inheritance of mitochondrial genome in bananas (Musa acuminata). Curr. Genet. 25, 342-345.

Gao, M., Li, G., Yang, B., McCombie, W. R., and Quiros, C. F. (2004) Comparative analysis of a Brassica BAC clone containing several major aliphatic glucosinolate genes with its corresponding Arabidopsis sequence. Genome 47, 666-679.

Gao, M., Li, G., McCombie, W. R., and Quiros, C. F. (2005) Comparative analysis of a transposon-rich Brassica oleracea BAC clone with its corresponding sequence in $A$. thaliana. Theor. Appl. Genet. 111, 949-955.

Gao, M., Li, G., Potter, D., McCombie, W. R., and Quiros, C. F. (2006) Comparative analysis of methylthioalkylmalate synthase (MAM) gene family and flanking DNA sequences in Brassica oleracea and A. thaliana. Plant Cell Rep. 25, 592-598.

Handa, H. (2007) Investigation of the origin and transmission of linear mitochondrial plasmid based on phylogenetic analysis in Japanese rapeseed varieties. Genome 50, 234-240.

Handa, H. (2008) Liner plasmid s in plant mitochondria: Peaceful coexistences or malicious invasions? Mitochondrion 8, $15-25$.

Handa, H., Ohkawa, Y., and Nakajima, K. (1990) Mitochondrial genome of rapeseed (Brassica napus L.). 1. Interspesific variation of mitochondrial DNA. Jpn. J. Genet. 65, 17-24.

Handa, H., Itani, K., and Sato, H. (2002) Structural features and expression analysis of a liner mitochondrial plasmid in rapeseed (Brassica napus L.). Mol. Genet. Genomics 267, 797805 .
Hatakeyama, K., Horisaki, A., Niikura, S., Narusaka, Y., Abe, H., Yoshiaki, H., Ishida, M., Fukuoka, H., and Matsumoto, S. (2010) Mapping of quantitative trait loci for high level of self-incompatibility in Brassica rapa L. Genome 53, 257265.

Havey, M. J. (1997) Predominant paternal transmission of the mitochondrial genome in cucumber. J. Hered. 88, 232-235.

Hori, K., Kobayashi, T., Shimizu, A., Sato, K., Takeda, K., and Kawasaki, S. (2003) Efficient construction of high-density linkage map and its application to QTL analysis in barley. Theor. Appl. Genet. 107, 806-813.

Ibrahim, N., Handa, H., Cosset, A., Koulintchenko, M., Konstantinov, Y., Lightowlers, N. R., Dietrich, A., and Weber-Lotfi, F. (2011) DNA delivery to mitochondria: sequence specificity and energy enhancement. Pharm. Res. 28, 2871-2882.

Iniguez-Luy, F. L., Voort, A. V., and Osborn, T. C. (2008) Development of a set of public SSR markers derived from genomic sequence of a rapid cycling Brassica oleracea L. genotype. Theor. Appl. Genet. 117, 977-985.

Iwata, H., and Ninomiya, S. (2006) AntMap: Constructing genetic linkage maps using an ant colony optimization algorithm. Breed. Sci. 56, 371-377.

Kakizaki, T., Kato, T., Fukino, N., Ishida, M., Hatakeyama, K., and Matsumoto, S. (2011) Identification of quantitative trait loci controlling late bolting in Chinese cabbage (Brassica rapa L.) parental line Nou 6 gou. Breed. Sci. 61, 151-159.

Koulintchenko, M., Konstantinov, Y., and Dietrich, A. (2003) Plant mitochondria actively import DNA via the permeability transition pore complex. EMBO J. 22, 1245-1254.

Koulintchenko, M., Temperley, R. J., Mason, P. A., Dietrich, A., and Lightowlers, R. N. (2006) Natural competence of mammalian mitochondria allows the molecular investigation of mitochondrial gene expression. Hum. Mol. Genet. 15, 143154.

Kresovich, S., Szewc-McFadden, A. K., Bliek, S. M., and McFerson, J. R. (1995) Abundance and characterization of simplesequence repeats (SSRs) isolated from a size-fractionated genomic library of Brassica napus L. (rapeseed). Theor. Appl. Genet. 91, 206-211.

Kuroiwa, T., Kawazu, T., Utida, H., Ohta, T., and Kuroiwa, H. (1993) Direct evidence of plastid DNA and mitochondrial DNA in sperm cells in relation to bi- parental inheritance of organelle DNA in Pelargonium zonale by fluo- rescence/electron microscopy. Eur. J. Cell Biol. 62, 307-313.

Li, H., Chen, X., Yang, Y., Xu, J., Gu, J., Fu, J., Qian, X., Zhang, S., Wu, J., and Liu, K. (2010) Development and genetic mapping of microsatellite markers from whole genome shotgun sequences in Brassica oleracea. Mol. Breed. DOI: 10.1007/ s11032-010-9509-y.

Logan, D. C. (2006) Plant mitochondrial dynamics. Biochim. Biophys. Acta 1763, 430-441.

Logan, D. C. (2010) Mitochondrial fusion, division and positioning in plants. Biochem. Soc. Trans. 38, 789-795.

Matsushima, R., Hamamura, Y., Higashiyama, T., Arimura, S., Sodmergen, Tsutsumi, N., and Sakamoto, W. (2008) Mitochondrial dynamics in plant male gametophyte visualized by fluorescent live imaging. Plant Cell Physiol. 49, 81-91.

Matsushima, R., Tang, L. Y., Zhang, L., Yamada, H., Twell, D., and Sakamoto, W. (2011) A conserved, $\mathrm{Mg}^{2+}$-dependent exonuclease degrades organelle DNA during Arabidopsis pollen development. Plant Cell 23, 1608-1624.

Murray, M. G., and Thompson, W. F. (1980) Rapid isolation of high molecular weight plant DNA. Nucleic Acids Res. 8, $4321-4325$. 
Nagata, N. (2010) Mechanisms for independent cytoplasmic inheritance of mitochondria and plastids in angiosperms. J. Plant Res. 123, 193-199.

Nagata, N., Saito, C., Sakai, A., Kuroiwa, H., and Kuroiwa, T. (1999) The selective increase or decrease of organellar DNA in generative cells just after pollen mitosis one controls cytoplasmic inheritance. Planta 209, 53-65.

Piquemal, J., Cinquin, E., Couton, F., Rondeau, C., and Seignoret, E. (2005) Construction of an oilseed rape (Brassica napus L.) genetic map with SSR markers. Theor. Appl. Genet. 111, 1514-1523.

Preuten, T., Cincu, E., Fuchs, J., Zoschke, R., Liere, K., and Börner, T. (2010) Fewer genes than organelles: extremely low and variable gene copy numbers in mitochondria of somatic plant cells. Plant J. 64, 948-959.

Radoev, M., Becker, H. C., and Ecke, W. (2008) Genetic analysis of heterosis for yield and yield components in rapeseed (Brassica napus L.) by quantitative trait locus mapping. Genetics 179, 1547-1558.

Rozen, S., and Skaletsky, H. J. (2000) Primer3 on the WWW for general users and for biologist programmers. In: Bioinformatics Methods and Protocols: Methods in Molecular Biology (eds.: S. Krawetz, and S. Misener), pp. 365-386. Humana Press, Totowa, NJ.

Sakurai, R., Nomura, H., Moriyama, Y., and Kawano, S. (2004) The mitochondrial plasmid of the true slime mold Physarum polycephalum bypasses uniparental inheritance by promoting mitochondrial fusion. Curr. Genet. 46, 103-114.

Sato, H., Saito, C., and Handa, H. (2004) Mitochondrial DNA is decreased during the pollen development of rapeseed (Brassica napus L.), but RNA polymerase encoded by the mitochondrial liner plasmid still exists in the mature pollen. Protoplasma 224, 179-185.

Saumitou-Laprade, P., Pannebecker, G., Maggouta, F., Jean, R., and Mchaelis, G. (1989) A liner $10.4 \mathrm{~kb}$ Plasmid in the mitochondria of Beta maritima. Curr. Genet. 16, 181-186.

Shedge, V., Arrieta-Montiel, M., Christensen, A. C., and Mackenzie, S. A. (2007) Plant mitochondrial recombination surveillance requires unusual $\operatorname{Rec} A$ and $M u t S$ homologs. Plant Cell 19, 1251-1264.

Stieneke, D. L., and Eujayl, I. A. (2007) Imperfect SSR Finder. http://ssr.nwisrl.ars.usda.gov/. Version 1.0. Kimberly, ID: USDA-ARS-NWISRL

Suwabe, K., Tsukazaki, H., Iketani, H., Hatakeyama, K., Kondo, M., Fujimura, M., Nunome, T., Fukuoka, H., Hirai, M., and Matsumoto, S. (2006) Simple sequence repeat-based comparative genomics between Brassica rapa and Arabidopsis thaliana: origin of clubroot resistance. Genetics 173, 309319.

Szewc-McFadden, A. K., Kresovich, S., Bliek, S. M., Mitchell, S. E., and McFerson, J. R. (1996) Identification of polymorphic, conserved simple sequence repeats (SSRs) in cultivated Brassica species. Theor. Appl. Genet. 93, 534-538.

Trusty, J. L., Johnson, K. J., Lockaby, B. G., and Goertzenm, L. R. (2007) Bi-parental cytoplasmic DNA inheritance in Wisteria (Fabaceae): evidence from a natural experiment. Plant Cell Physiol. 48, 662-665.

Voorrips, R. E. (2002) MapChart: Software for the Graphical Presentation of Linkage Maps and QTLs. J. Hered. 93, 7778.

Wang, S., Basten, C. J., and Zeng, Z. B. (2007) Windows QTL cartographer 2.5. Department of Statistics, North Carolina State University, Raleigh, NC. http://statgen.ncsu.edu/ qtlcart/WQTLCart.htm.

Xu, J., Qian, X., Wang, X., Li, R., Cheng, X., Yang, Y., Fu, J., Zhang, S., King, G.J., Wu, J., and Liu, K. (2010) Construction of an integrated genetic linkage map for the A genome of Brassica napus using SSR markers derived from sequenced BACs in B. rapa. BMC Genomics 11, 594-608.

Yu, H. S., Huang, B. Q., and Russell, S. D. (1994) Transmission of male cytoplasm during fertilization in Nicotiana tabacum. Sex. Plant Reprod. 7, 313-323.

Zaegel, V., Guermann, B., Le Ret, M., Andres, C., and Meyer, D. (2006) The plant-specific ssDNA binding protein OSB1 is involved in the stoichiometric transmission of mitochondrial DNA in Arabidopsis. Plant Cell 18, 3548-3563. 